\title{
Knockdown of circDENND4C inhibits glycolysis, migration and invasion by up- regulating miR-200b/c in breast cancer under hypoxia
}

Shasha Ren ${ }^{1 \dagger}$, Jiuzhou Liu ${ }^{1+}$, Yun Feng ${ }^{1}$, Zhenyu Li ${ }^{1}$, Liang He${ }^{1}$, Leilei $\mathrm{Li}^{1}$, Xiaozhong Cao ${ }^{1}$, Zhenghua Wang ${ }^{1}$ and Yanwu Zhang ${ }^{2 *}$

\begin{abstract}
Background: Hypoxia is a key feature of breast cancer, which affects cancer development, metastasis and metabolism. Previous studies suggested that circular RNAs (circRNAs) could participate in cancer progression and hypoxia regulation. This study aimed to investigate the role of circRNA differentially expressed in normal cells and neoplasia domain containing 4C (circDENND4C) in breast cancer progression under hypoxia.
\end{abstract}

Methods: Forty-three patients with breast cancer were involved in this study. Breast cancer cell lines MDA-MB-453 and SK-BR-3 were cultured under hypoxia $\left(1 \% \mathrm{O}_{2}\right)$ for experiments in vitro. The expression levels of circDENND4C, microRNA-200b (miR-200b) and miR-200c were measured by quantitative real-time polymerase chain reaction. Glycolysis was investigated by glucose consumption, lactate production and hexokinase II (HK2) protein level. Migration and invasion were evaluated via trans-well assay and protein levels of matrix metallopeptidase 9 (MMP9) and MMP2. The interaction between circDENND4C and miR-200b or miR-200c was explored by bioinformatics analysis, luciferase assay and RNA immunoprecipitation. Murine xenograft model was established to investigate the anti-cancer role of circDENND4C in vivo.

Results: circDENND4C highly expressed in breast cancer was up-regulated in response to hypoxia. Knockdown of circDENND4C decreased glycolysis, migration and invasion in breast cancer cells under hypoxia. circDENND4C was validated as a sponge of miR-200b and miR-200c. Deficiency of miR-200b or miR-200c reversed the suppressive effect of circDENND4C knockdown on breast cancer progression. Moreover, silence of circDENND4C reduced xenograft tumor growth by increasing miR-200b and miR-200c.

Conclusion: circDENND4C silence suppresses glycolysis, migration and invasion in breast cancer cells under hypoxia by increasing miR-200b and miR-200c.

Keywords: Breast cancer, circDENND4C, miR-200b, miR-200c, Glycolysis, Migration

\footnotetext{
*Correspondence: e4812207xiex@163.com

†'Shasha Ren and Jiuzhou Liu co-first authors

${ }^{2}$ Department of Breast Surgery, the Third Affiliated Hospital of Zhengzhou

University, No. 7 Kangfuqian Street, Erqi District, Zhengzhou 450052, China

Full list of author information is available at the end of the article
}

(c) The Author(s). 2019 Open Access This article is distributed under the terms of the Creative Commons Attribution 4.0 International License (http://creativecommons.org/licenses/by/4.0/), which permits unrestricted use, distribution, and reproduction in any medium, provided you give appropriate credit to the original author(s) and the source, provide a link to the Creative Commons license, and indicate if changes were made. The Creative Commons Public Domain Dedication waiver (http://creativecommons.org/publicdomain/zero/1.0/) applies to the data made available in this article, unless otherwise stated. 


\section{Background}

Breast cancer is the most common malignancy with leading cause of death from cancer in women worldwide [1]. Hypoxia is an important feature of solid tumors, which contributes to progression of breast cancer and increases the risk of metastasis and mortality [2]. Under condition of hypoxia, glycolysis could maintain survival of cancer cells and promote cell progresses, such as proliferation, migration and invasion [3]. Hence, it is urgent to explore new mechanism underlying breast cancer progression under hypoxia.

Circular RNAs (circRNAs) are a class of single-stranded noncoding RNA molecules, which play essential roles in development and diagnosis of human diseases and cancers $[4,5]$. Moreover, circRNAs have been regarded as potential therapeutic targets in breast cancer [6]. Xu et al. reported that circRNA transcriptional adaptor 2A (circTADA2A) inhibits cell proliferation, migration and invasion by regulating microRNA-203a-3p/suppressor of cytokine signaling 3 (SOCS3) axis in breast cancer [7]. Furthermore, Zhang et al. suggested that circRNA has circ_0052112, generated from zinc finger 83, could promote cell migration and invasion by sponging microRNA-125a-5p in breast cancer [8]. CircRNA differentially expressed in normal cells and neoplasia domain containing $4 \mathrm{C}$ (circDENND4C) is a hypoxiaassociated RNA molecule [9]. Notably, previous study indicated that high expression of circDENND4C promotes cell proliferation in breast cancer under hypoxia condition [10]. However, the role and mechanism of circDENND4C in breast cancer progression remain largely unknown.

CircRNAs usually exhibit their biological roles by acting as microRNA (miRNA) inhibitors or sponges by binding the seed sites to quench the normal function of miRNA in human cancers [11]. miRNAs are a class of small noncoding RNAs with 18-25 nucleotides, which play as promising targets for diagnosis, prognosis and therapeutics of breast cancer [12]. Previous works have demonstrated that miR-200b and miR-200c could serve as important tumor suppressor by inhibiting cell proliferation, migration and invasion in breast cancer [13-17]. More importantly, the complementary sites between circDENND4C and miR-200b or miR-200c predicted by bioinformatics analysis using starBase stimulated us to hypothesize that miR-200b and miR-200c might be required for circDENND4C-mediated progression of breast cancer.

In the current study, we measured the expression level of circDENND4C and investigated its biological role in glycolysis, migration and invasion in breast cancer cells under hypoxia. Moreover, we explored whether the regulatory mechanism was associated with miR-200b and miR-200c.

\section{Materials and methods}

\section{Patients and tissues}

A total of 43 patients with breast cancer were recruited from the Third Affiliated Hospital of Zhengzhou University. All participants have provided the written informed consents. The clinical features of patients were summarized in Table 1. The paired tumor tissues and corresponding adjacent normal samples were obtained prior to any other treatment and stored at $-80{ }^{\circ} \mathrm{C}$ until used. The protocol of this study was approved by the ethics committee of the Third Affiliated Hospital of Zhengzhou University.

\section{Cell culture, hypoxia stimulation and cell transfection}

Breast cancer cell lines (MDA-MB-453 and SK-BR-3) and normal human breast epithelial cells MCF-10A were purchased from the BeNa Culture Collection (Beijing, China), and cultured in RPMI-1640 medium (Gibco, Carlsbad, CA, USA) plus 10\% fetal bovine serum at $37{ }^{\circ} \mathrm{C}$ with $5 \% \mathrm{CO}_{2}$. For hypoxia stimulation, MDA-MB-453 and SK-BR-3 cells were growth in a hypoxia chamber with $1 \% \mathrm{O}_{2}$ for various exposure times $(0$, $3,6,12,24$ and $48 \mathrm{~h}$ ).

Table 1 Association between circDENND4C and the clinicopathological characteristics of breast cancer

\begin{tabular}{|c|c|c|c|c|}
\hline & \multirow[t]{2}{*}{$N(\%)$} & \multicolumn{2}{|c|}{ circDENND4C level } & \multirow[t]{2}{*}{$P$ value } \\
\hline & & High (\%) & Low (\%) & \\
\hline \multicolumn{4}{|l|}{ Age(years) } & $P>0.05$ \\
\hline$\geq 55$ & $24(55.8)$ & $13(54.2)$ & $11(45.8)$ & \\
\hline$<55$ & $19(44.2)$ & $10(52.6)$ & $9(47.4)$ & \\
\hline \multicolumn{4}{|l|}{ Menopause } & $P>0.05$ \\
\hline No & $26(60.5)$ & $14(53.8)$ & $12(46.2)$ & \\
\hline Yes & $17(39.5)$ & $9(52.9)$ & $8(47.1)$ & \\
\hline \multicolumn{4}{|l|}{ TNM stage } & $P<0.05$ \\
\hline$|-| \mid$ & $23(53.5)$ & $8(34.8)$ & $15(65.2)$ & \\
\hline III-IV & $20(46.5)$ & $15(75.0)$ & $5(25.0)$ & \\
\hline \multicolumn{4}{|c|}{ Lymph node metastasis } & $P<0.05$ \\
\hline No & $22(51.2)$ & $8(36.4)$ & $14(63.6)$ & \\
\hline Yes & $21(48.8)$ & $15(71.4)$ & $6(28.6)$ & \\
\hline \multicolumn{4}{|l|}{ Tumor size } & $P<0.05$ \\
\hline$\geq 2 \mathrm{~cm}$ & $25(58.1)$ & $17(68.0)$ & $8(32.0)$ & \\
\hline$<2 \mathrm{~cm}$ & $18(41.9)$ & $6(33.3)$ & $12(66.7)$ & \\
\hline \multicolumn{4}{|l|}{ Subtype } & $P>0.05$ \\
\hline TNBC & $19(44.2)$ & $10(52.6)$ & $9(47.4)$ & \\
\hline HER2 & $5(11.6)$ & $3(60.0)$ & $2(40.0)$ & \\
\hline Luminal A & $8(18.6)$ & $4(50)$ & $4(50)$ & \\
\hline Luminal B & 11 (25.6) & $6(54.5)$ & $5(45.5)$ & \\
\hline
\end{tabular}

Abbreviations: TNM tumor node metastasis, TNBC triple negative breast cancer HER2 human epidermal growth factor receptor 2 
Small interfering RNA (siRNA) against circDENND4C (si-circ) (5'-AAGUAGCACUGCUCUUCAAAA-3'), siRNA negative control (si-NC) (5'-UCUCCGAACGUGUCACGU TT-3'), pcDNA-based circDENND4C overexpression vector (circ), pcDNA vector, miR-200b mimic (miR-200b) (5'-UAAUACUGCCUGGUAAUGAUGA-3'), miR-200c mimic (miR-200c) (5'-UAAUACUGCCGGGUAAUG AUGGA-3'), mimic negative control (miR-NC) (5'UUCUCCGAACGUGUCACGUTT-3'), miR-200b inhibitor (anti-miR-200b) (5'-UCAUCAUUACCAGGCAGU AUUA-3'), miR-200c inhibitor (anti-miR-200c) (5' UCCAUCAUUACCCGGCAGUAUUA-3') and inhibitor negative control (anti-miR-NC) (5'-UUCUCCGAAC GUGUCACGUTT-3') were generated from Genepharma (Shanghai, China). When reaching 60\% confluence, MDA-MB-453 and SK-BR-3 cells were transfected with $30 \mathrm{nM}$ oligonucleotides or $200 \mathrm{ng}$ vector using Lipofectamine 3000 (Invitrogen, Carlsbad, CA, USA) following the manufactures' instructions. After transfection for 24h, MDA-MB-453 and SK-BR-3 cells were collected for subsequent analyses.

\section{Quantitative real-time polymerase chain reaction (qRT- PCR)}

Trizol reagent (Thermo Fisher Scientific, Waltham, MA USA) was used for RNA isolation from tissues or cells following the manufacturer's protocols. For detecting level of circDENND4C, total RNA was treated by RNase R (Geneseed, Guangzhou, China) to improve purity of circRNA. The miScript Reverse Transcription Kit (Qiagen, Dusseldorf, Germany) was used for reverse transcription with 500 ng total RNA treated by RNase R or not and qRT-PCR was performed with the diluted cDNA products, special primers and SYBR Green mix (Thermo Fisher Scientific). The amplification conditions were: $95^{\circ} \mathrm{C}$ for $1 \mathrm{~min}, 40$ cycles of $95^{\circ} \mathrm{C}$ for $10 \mathrm{~s}$ and $60^{\circ} \mathrm{C}$ for $30 \mathrm{~s}$. The primers were as follows: circDENND4C (Forward, 5'-GGGGCAGCAGTATT GTGAAA-3'; Reverse, 5'-AAGACTGTGTGCTCCCCA TT-3'); $\quad \beta$-actin (Forward, 5'-TCATGAAGTGTGAC GTGGACATC-3'; Reverse, 5'-CAGGAGGAGCAATG ATCTTGATCT-3'); miR-200b (Forward, 5'-GCGGCT AATACTGCCTGGTAA-3'; Reverse, 5' - GTGCAGGGTC CGAGGT-3'); miR-200c (Forward, 5'-TAATACTGCC GGGTAATGATGGA-3'; Reverse, 5'-CCAGTGCAGG GTCCGAGGT-3'); U6 (Forward, 5' -CTCGCTTCGG CAGCACA-3'; Reverse, 5'-AACGCTTCACGAATTTGC GT-3'). The relative expression levels of circDENND4C, miR-200b and miR-200c were determined with $\beta$-actin and $\mathrm{U} 6$ as internal control respectively by $2^{-\Delta \Delta \mathrm{Ct}}$ method [18].

\section{Glucose consumption and lactate production}

Transfected or non-transfected MDA-MB-453 and SKBR-3 cells $\left(1 \times 10^{5} /\right.$ well $)$ were seeded into 6 -well plates overnight and then incubated in hypoxia or normoxia condition for $48 \mathrm{~h}$. Glucose Assay Kit and Lactate Assay Kit (Sigma, St. Louis, MO, USA) were used for detection of glucose consumption and lactate production respectively following the manufacturer's protocol. The relative levels of glucose consumption and lactate production in all treated groups were normalized to normoxia group.

\section{Western blot}

After the indicated treatment, MDA-MB-453 and SKBR-3 cells were collected and lysed in RIPA lysis buffer (Beyotime, Shanghai, China). The lysates were centrifuged at $12,000 \mathrm{~g}$ for $10 \mathrm{~min}$ and then total protein in supernatant was qualitied by using BCA protein assay kit (Beyotime). Following the boiled water bath, the equal amounts of proteins $(30 \mu \mathrm{g})$ were loaded on SDSPAGE gel and transferred to PVDF membranes (Millipore, Billerica, MA, USA). After blocking the non-specific binding sites with 5\% non-fat milk, the membranes were probed with antibodies against hexokinase II (HK2) (ab227198, 1:5000, $102 \mathrm{kDa}$ ), matrix metallopeptidase 9 (MMP9) (ab38898, 1:1000, $92 \mathrm{kDa}$ ), MMP2 (ab97779, 1: $1000,72 \mathrm{kDa}$ ) or $\beta$-actin (ab8227, 1:3000, $42 \mathrm{kDa}$ ) overnight at $4{ }^{\circ} \mathrm{C}$, along with horseradish peroxidase-labeled IgG (ab6721, 1:10000) for $2 \mathrm{~h}$ at room temperature. The antibodies used in this study were purchased from Abcam (Cambridge, MA, USA). The blots were visualized using BeyoECL Plus (Beyotime) and the relative protein level with $\beta$-actin as an endogenous control was normalized to normoxia group.

\section{Trans-well assay}

For migration and invasion assays, trans-well assay was performed using 24-well trans-well chamber pre-coated with or without Matrigel (BD Bioscience, San Jose, CA, USA). Treated MDA-MB-453 and SK-BR-3 cells $(4 \times$ $10^{4} /$ well) in serum-free RPMI-1640 medium were added to the upper chamber and $10 \%$ fetal bovine serum medium was added in the lower chamber. Cells were cultured at $37^{\circ} \mathrm{C}$ with $5 \% \mathrm{CO}_{2}$ for $24 \mathrm{~h}$ and then those on the lower surface were fixed with methanol (Sigma) and stained with $1 \%$ crystal violet (Sigma). A 200X magnification microscope (Olympus, Tokyo, Japan) was used to photograph the migrated and invasive cells with three randomly selected fields.

\section{Bioinformatics analysis, luciferase assay and RNA immunoprecipitation (RIP)}

Bioinformatics analysis predicted the binding sites of circDENND4C and miR-200b or miR-200c by using starBase. The sequences of circDENND4C containing the predicted complementary sites of miR-200b or miR-200c (GCAGUA UU) at chr9: 1934268-1,934,275 were inserted into pmirGLO vectors (Promega, Madison, WI, USA) to generate wild-type luciferase reporter vector circDENND4C-WT 

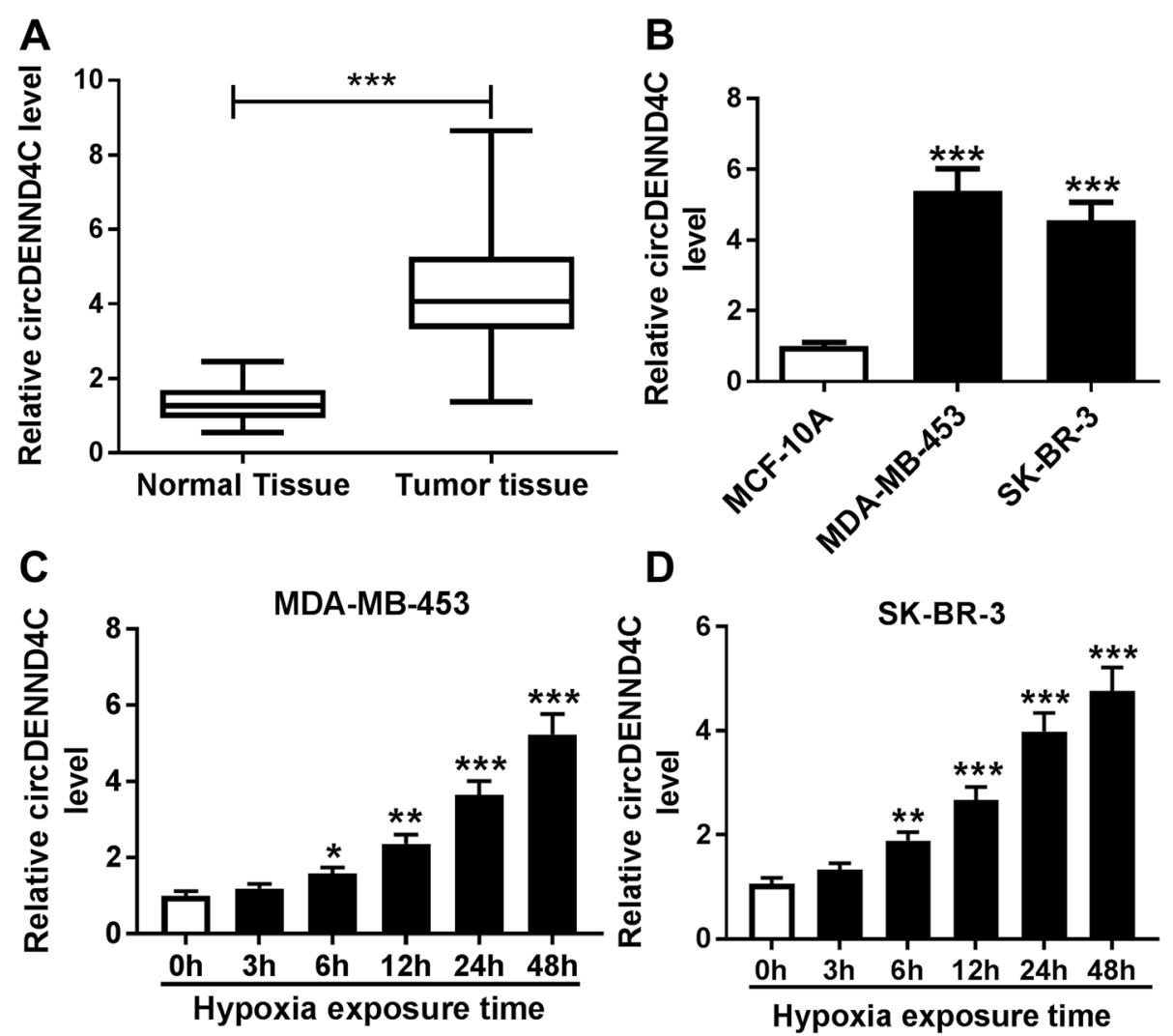

Fig. 1 The expression of circDENND4C is enhanced in breast cancer under hypoxia. a circDENND4C expression in breast cancer tissues and adjacent normal samples was measured by qRT-PCR. $n=43$. b The level of circDENND4C was detected in breast cancer cells (MDA-MB-453 and SK-BR-3) and normal cells (MCF-10A) by qRT-PCR. $\mathbf{c}$ and $\mathbf{d}$ The abundance of circDENND4C was examined in MDA-MB-453 and SK-BR-3 cells after hypoxia exposure for various time by qRT-PCR. ${ }^{*} P<0.05,{ }^{*} P<0.01$, ${ }^{* *} P<0.001$

(circ-WT). The corresponding mutant was generated by mutating the seed sites to AUGAGCAG, named as circDENND4C-MUT (circ-MUT). Luciferase assay was performed in MDA-MB-453 and SK-BR-3 cells co-transfected with $200 \mathrm{ng}$ circ-WT or circ-MUT and $30 \mathrm{nM}$ miR-200b, miR-200c or miR-NC using Lipofectamine 3000. A luciferase reporter assay kit (Promega) was used for the luciferase activity analysis at $24 \mathrm{~h}$ after the transfection.

For RIP assay, MDA-MB-453 and SK-BR-3 cells transfected with miR-200b, miR-200c or miR-NC were lysed in RIP lysis buffer. The combination of circDENND4C and miR-200b or miR-200c was examined by using the Magna RNA immunoprecipitation kit (Millipore) according to the manufacturer's instructions. The magnetic beads were pre-coated by antibody against Ago2 (ab32381, Abcam) or IgG (AP112, Sigma). The level of circDENND4C (circ) enriched by RIP was measured by qRT-PCR.

\section{Murine xenograft model}

The lentiviral vectors with sh-circDENND4C (sh-circ) or corresponding control (sh-NC) were constructed by FulenGen (Guangzhou, China). MDA-MB-453 cells were infected with sh-circ or sh-NC for $6 \mathrm{~h}$ and then stably transfected cells were selected under fluorescence microscope (Olympus) and flow cytometry (BD Bioscience). Five-week-old female BALB/c nude mice (Vital River Laboratory Animal Technology, Beijing, China) were injected subcutaneously with stably transfected MDA-MB-453 cells $\left(5 \times 10^{6}\right)$, termed as sh-circ or sh-NC group ( $n=3$ per group). The mice injected with non-transfected cells were classified as empty group. Tumor volume was monitored every week and calculated using the formula: volume $\left(\mathrm{mm}^{3}\right)=$ width $^{2} \times$ length/2. After 5 weeks following the inoculation, the mice were killed and tumor samples were weighted and harvested for measurement of circDENND4C, miR$200 \mathrm{~b}$ and miR-200c expression levels. The experiment was permitted by the Animal Research Committee of the Third Affiliated Hospital of Zhengzhou University and performed in accordance with the guidelines of use of laboratory animals.

\section{Statistical analysis}

GraphPad Prism 7 software (GraphPad Inc., La Jolla, CA, USA) was used for statistical analyses. Data from three 


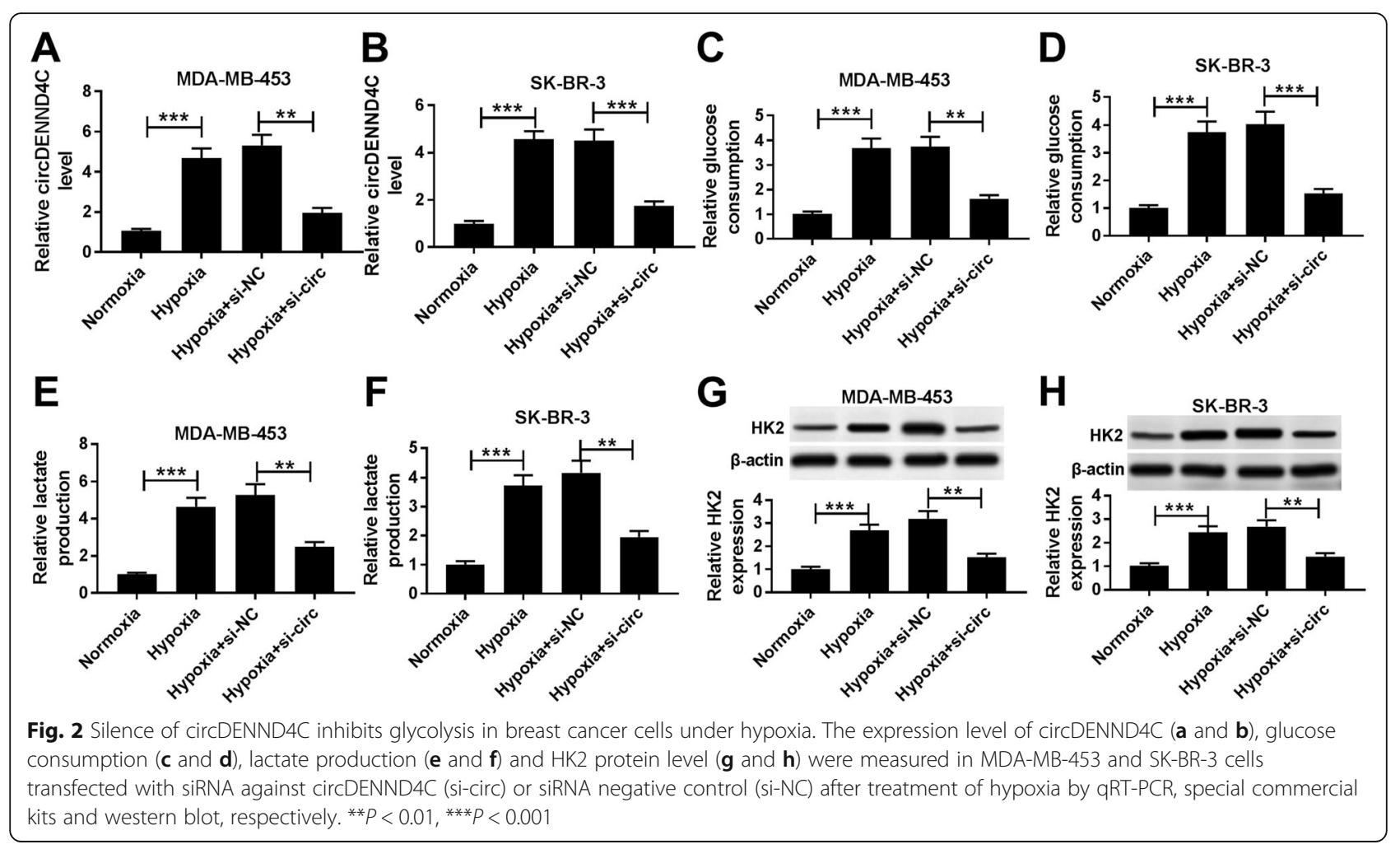

independent experiments were expressed as mean \pm standard deviation (S.D.). The comparison between two or more groups was performed by using paired student's $t$ test or one-way ANOVA with Tukey's post hoc test. The association between circDENND4C level and clinicopathologic features of breast cancer patients was analyzed by $X^{2}$ test. ${ }^{*} P<0.05,{ }^{* *} P<0.01$ and ${ }^{* * *} P<0.001$ were considered significant.

\section{Results}

The expression of circDENND4C is increased in breast cancer cells under hypoxia

To explore the role of circDENND4C (the scheme of circDENND4C was shown in Additional file 1: Figure. S1) in breast cancer, its expression was measured in breast cancer tissues and cells. The pathologies of tumor tissues and normal samples were displayed in Additional file 2: Figure S2. As shown in Fig. 1a and Additional file 3: Figure S3A, compared with corresponding adjacent normal tissues, tumor tissues $(n=$ 43) displayed significantly increased level of circDENND4C. More specifically, circDENND4C level was significantly increased in the four subtypes of breast cancer (TNBC, HER2, Luminal A and B) compared with that in normal tissues (Additional file 4: Figure S4). Moreover, by dividing into two groups according to the mean value of circDENND4C level, the high expression of circDENND4C was associated with tumor node metastasis stage, lymph node metastasis and tumor size $(P<0.05)$ but not with age, menopause and subtype $(P>0.05)$ (Table 1). In addition, the expression of circDENND4C was markedly enhanced in breast cancer cells (MDAMB-453 and SK-BR-3) compared with that in MCF-10A cells (Fig. 1b and Additional file 5: Figure S5). Furthermore, the expression of circDENND4C was detected in MDA-MB-453 and SK-BR-3 cells after exposure of hypoxia. Results showed that HIF1A (a hypoxia indicator) protein level was significantly increased in cells after treatment of hypoxia (Additional file 6: Figure S6A and $6 \mathrm{~B})$ and the abundance of circDENND4C was progressively enhanced in MDA-MB-453 and SK-BR-3 cells after exposure of hypoxia in a time dependent manner (Fig. 1c and $\mathrm{d}$ ). Besides, we confirmed the expression of circDENND4C was associated with HIF1A level in the two cell lines (Additional file 7: Figure S7A-7F).

Knockdown of circDENND4C inhibits glycolysis, migration and invasion in breast cancer cells under hypoxia

To investigate the effect of circDENND4C on breast cancer progression, its level was knocked down using si-circ in MDA-MB-453 and SK-BR-3 cells under hypoxia of 48 $\mathrm{h}$ (Fig. 2a and b). Moreover, treatment of hypoxia of $48 \mathrm{~h}$ induced obvious increase of glucose consumption, lactate production and HK2 protein level in MDA-MB-453 and SK-BR-3 cells, while silence of circDENND4C greatly weakened these events (Fig. 2c-h). Furthermore, the 


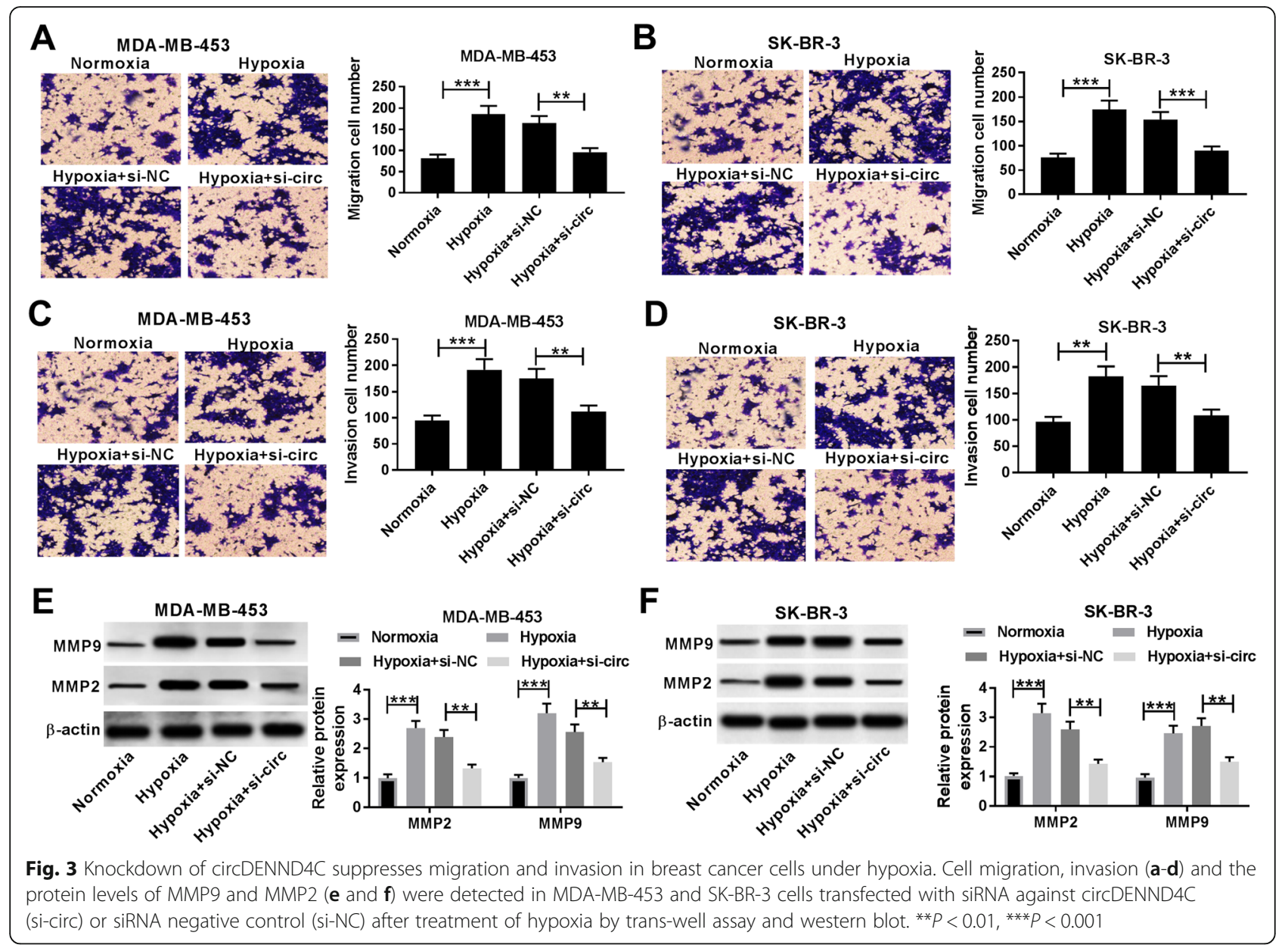

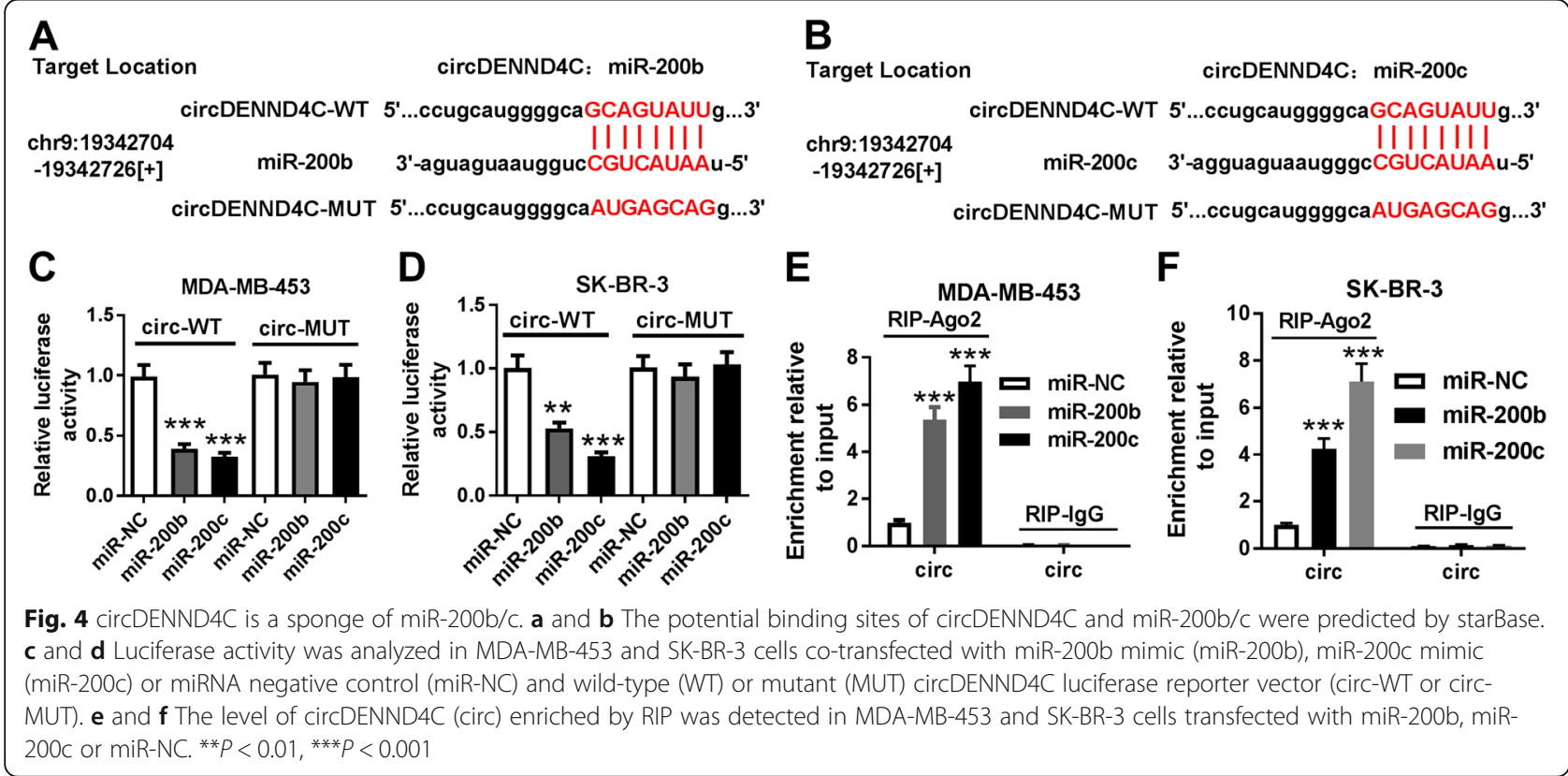




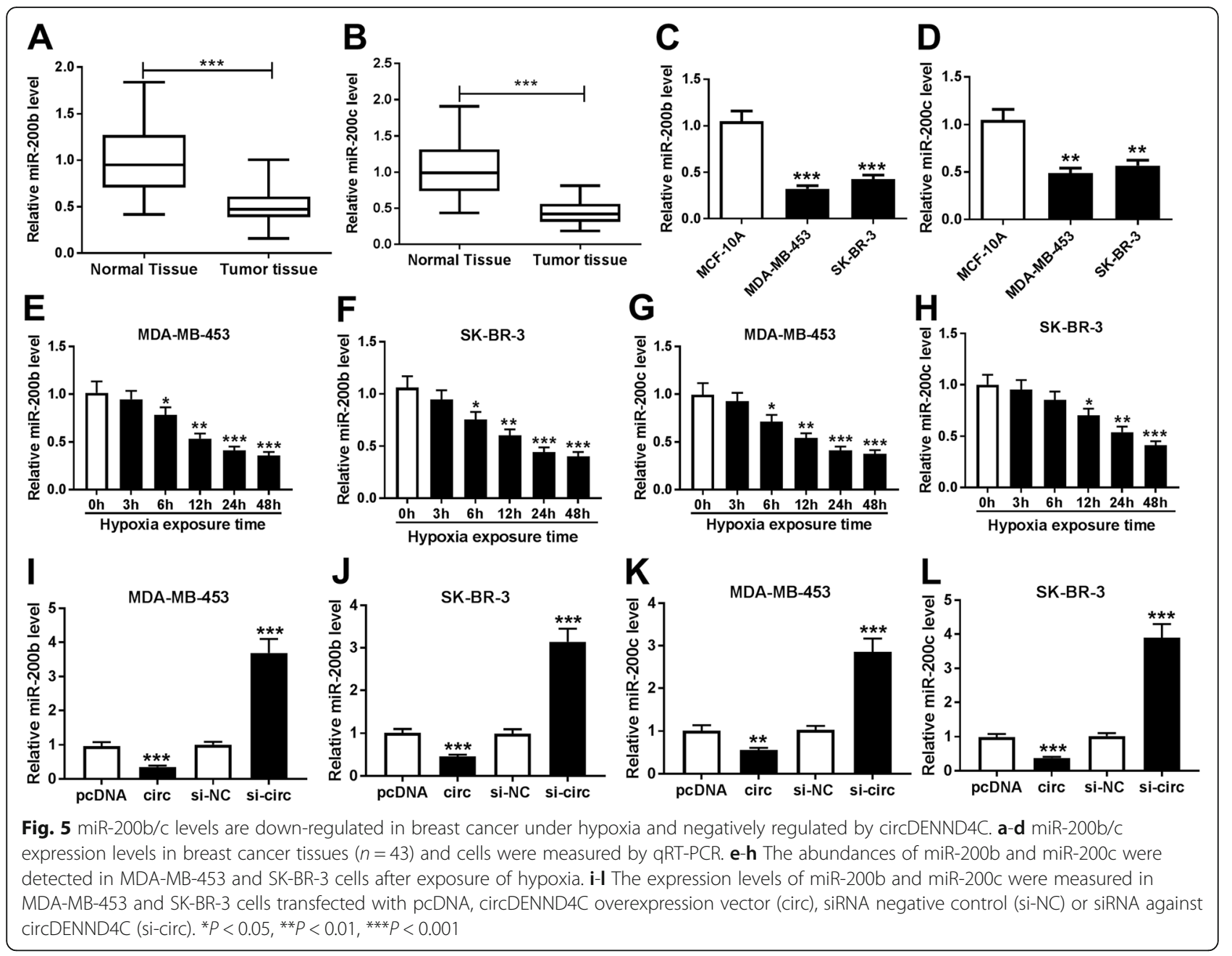

analyses of trans-well described that interference of circDENND4C notably decreased the abilities of cell migration and invasion promoted by hypoxia treatment in MDA-MB-453 and SK-BR-3 cells under hypoxia (Fig. 3ad). However, overexpression of circDENND4C showed little effect on migration and invasion of these two cell lines under normoxia (Additional file 8: Figure S8A-8C). In addition, the protein expression levels of MMP9 and MMP2 in the two cells were conspicuously elevated by hypoxia exposure, which was counteracted by silence of circDENND4C (Fig. 3e and $\mathrm{f}$ ).

\section{miR-200b and miR-200c are sponged by circDENND4C}

To elucidate the potential regulatory mechanism underlying circDENND4C in breast cancer progression, its target miRNAs were explored. As shown in Fig. 4a and $b$, bioinformatics analysis using starBase provided the predicted binding sites of circDENND4C and miR$200 \mathrm{~b}$ or miR-200c. To validate this prediction, luciferase assay and RIP assay were performed in MDA-MB453 and SK-BR-3 cells. As demonstrated in Fig. 4c and d, overexpression of miR-200b or miR-200c led to great loss of luciferase activity in circ-WT group, while they did not affect the activity in circ-MUT group. Furthermore, addition of miR-200b or miR-200c resulted in higher level of circDENND4C enriched by Ago2 RIP in MDA-MB-453 and SK-BR-3 cells than transfection of miR-NC, whereas IgG group showed little enrichment of circDENND4C (Fig. 4e and f).

miR-200b and miR-200c levels are decreased in breast cancer under hypoxia and negatively regulated by circDENND4C

To explore the role of miR-200b and miR-200c in breast cancer, their expression levels were detected. As shown in Fig. 5a and $\mathrm{b}$ and Additional file 3: Figure S3B and 3C, the expression levels of miR-200b and miR-200c were evidently reduced in breast cancer tissues $(n=43)$ compared with those in normal samples. Moreover, their levels were also decreased in MDA-MB-453 and SK-BR-3 cells in comparison to those in MCF-10A cells (Fig. $5 \mathrm{c}$ and d). In addition, after stimulation of hypoxia condition, the 


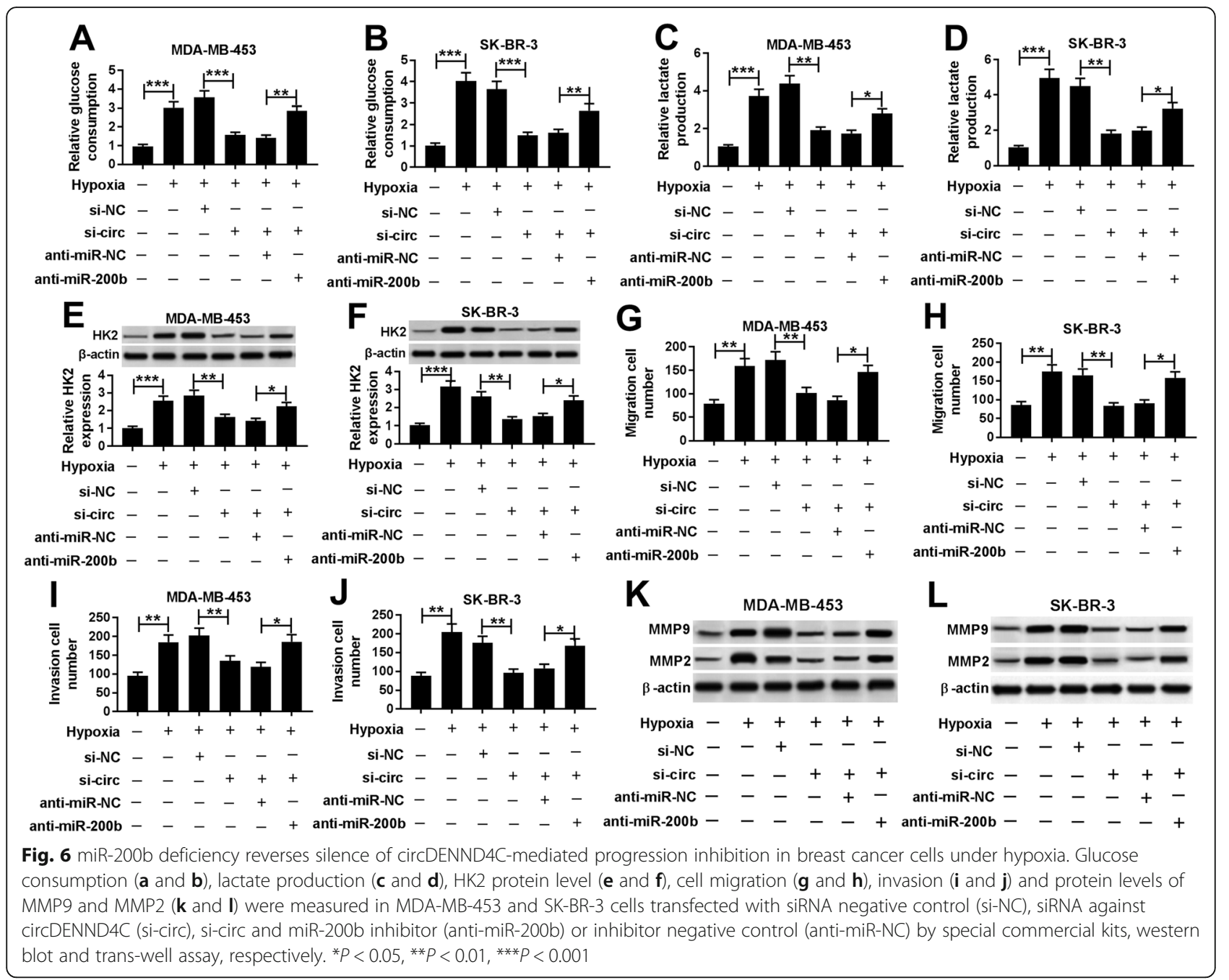

abundances of miR-200b and miR-200c were progressively down-regulated in the two cells in a time dependent manner (Fig. 5e-h). Besides, qRT-PCR assay also revealed that the expression levels of miR-200b and miR-200c in MDAMB-453 and SK-BR-3 cells were significantly decreased by overexpression of circDENND4C but increased via knockdown of circDENND4C (Fig. 5i-l).

\section{Knockdown of miR-200b or miR-200c reverses silence of} circDENND4C-induced progression inhibition in breast cancer cells under hypoxia

To explore whether miR-200b was responsible for circDENND4C-mediated breast cancer progression, MDAMB-453 and SK-BR-3 cells were transfected with si-NC, si-circ, si-circ and anti-miR-200b or anti-miR-NC before treatment of hypoxia. As shown in Fig. 6a-f, knockdown of miR-200b attenuated silence of circDENND4C-mediated inhibition of glucose consumption, lactate production and HK2 protein level in MDA-MB-453 and SK-BR-3 cells under hypoxia. Moreover, miR-200b deficiency alleviated the suppressive effect of circDENND4C knockdown on migration and invasion in the two cells under hypoxia (Fig. 6g-j). Additionally, the protein levels of MMP9 and MMP2 inhibited by circDENND4C interference were restored by miR-200b absence (Fig. 6k and l). Similarly, to explore whether circDENND4C-mediated breast cancer progression was modulated by miR-200c, MDA-MB-453 and SK-BR-3 cells were transfected with si-NC, si-circ, si-circ and anti-miR-200c or anti-miR-NC and then treated by hypoxia. Results showed that knockdown of miR-200c abated the inhibitive effect of circDENND4C silence on glucose consumption, lactate production, HK2 protein level, migration, invasion, MMP9 and MMP2 protein levels in MDA-MB-453 and SK-BR-3 cells under hypoxia (Fig. 7a-1).

Knockdown of circDENND4C decreases xenograft tumor growth by increasing miR-200b and miR-200c

To further investigate the anti-cancer role of circDENND4C silence, MDA-MB-453 cells stably transfected 


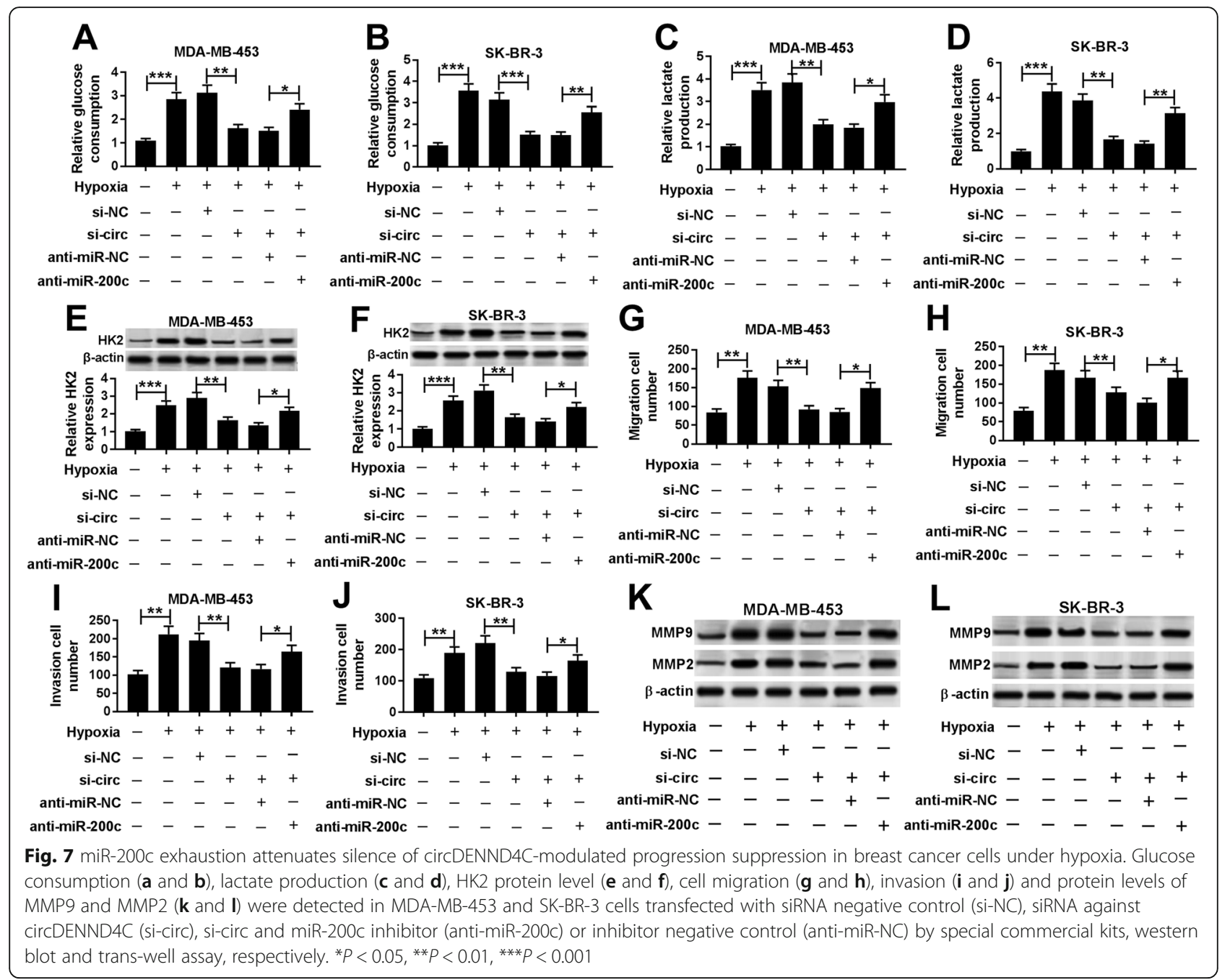

with sh-circ or sh-NC or non-transfected cells (Empty) were used to establish xenograft model in vivo. The infection efficacy was confirmed in Additional file 9: Figure S9A and 9B. After cell injection for 5 weeks, tumor volume and weight were significantly reduced in sh-circ group compared with those in sh-NC or empty group (Fig. 8a and b). Meanwhile, HIF1A expression was notably decreased in shcirc group compared with those in sh-NC or empty group (Additional file 10: Figure S10). Moreover, the expression of circDENND4C was decreased $48 \%$ in sh-circ group when compared with that in sh-NC group (Fig. 8c). However, the levels of miR-200b and miR-200c were increased 3.12-fold and 2.56-fold respectively in sh-circ group in comparison to those in sh-NC group (Fig. 8d and e).

\section{Discussion}

Hypoxia is a key feature of cancers and circRNAs are involved in regulation of hypoxia [9]. Among all breast cancer-associated with circRNA, circDENND4C is a circRNA in response to hypoxia. This study was the first to investigate the effect of circDENND4C on glycolysis, migration and invasion in breast cancer and explore the potential sponging miRNAs.

To resist hypoxia stress, cancer cell would trigger hypoxia-inducible factor 1 alpha (HIF1A) expression, which is related with dysregulation of circRNA. Previous study suggested that circDENND4C is a HIF1A-associated circRNA, which is highly expressed in hypoxia condition and promotes cell proliferation at 4 days in breast cancer [10]. Similarly, we also found that circDENND4C expression was enhanced in breast cancer cells after treatment of hypoxia. Moreover, high expression of circDENND4C predicted poor outcomes of patients with breast cancer. The hypoxia environment would lead to the reprogramming of glycolytic metabolism, which is characterized by glucose consumption and lactate production. Furthermore, HK2 is a key enzyme associated with glycolysis in cancers, including breast cancer [19-21]. Under hypoxia, glucose consumption, lactate production and HK2 protein level were enhanced in breast cancer cells, indicating that 

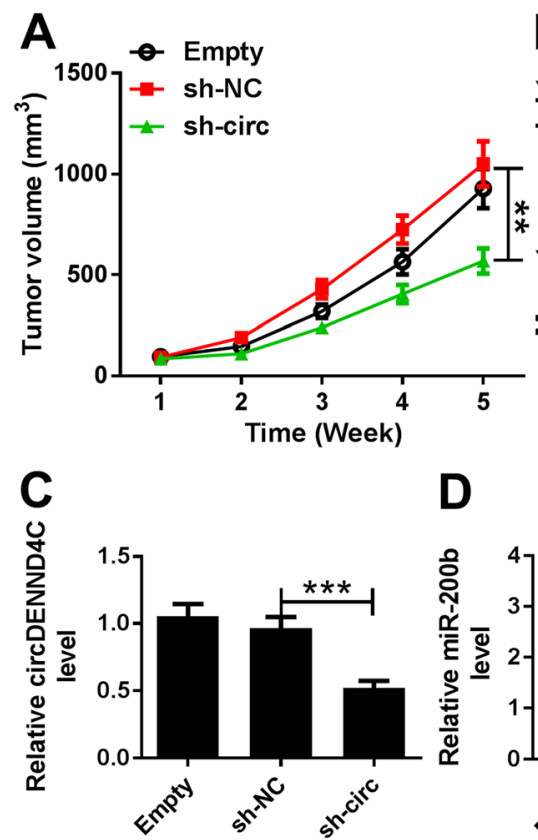
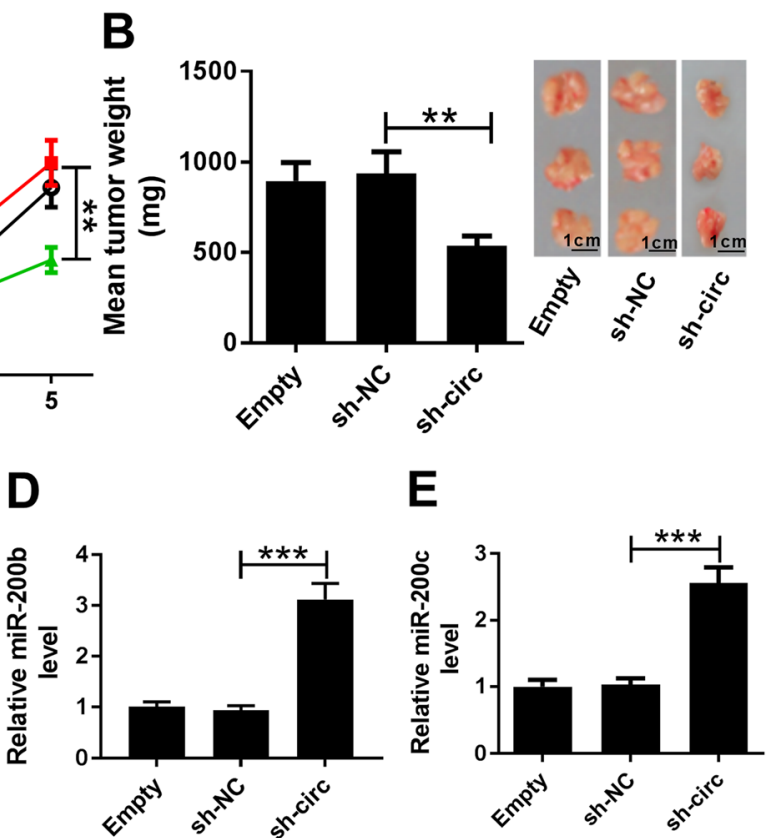

Fig. 8 Knockdown of circDENND4C decreases breast cancer cell xenograft tumor growth. $\mathbf{a}$ and $\mathbf{b}$ Tumor volume and weight were measured in shRNA against circDENND4C (sh-circ), shRNA negative control (sh-NC) or non-transfected (Empty) group. c-e The expression levels of circDENND4C, miR-200b and miR-200c were detected in tumor tissues by qRT-PCR. ${ }^{* *} P<0.01,{ }^{* * *} P<0.001$

glycolysis was triggered. However, loss-of-function experiments uncovered that circDENND4C knockdown could decrease glycolysis in breast cancer cells under hypoxia. Besides, hypoxia-induced epithelial mesenchymal transition is an important mechanism of tumor progression, which is responsible for cancer cell migration and invasion [22, 23]. MMPs, especially MMP9 and MMP2, are crucial biomarkers for migration and invasion in breast cancer [24-26]. By detecting MMPs levels and trans-well assay, we found that cell migration and invasion were increased in cells under hypoxia by increasing MMP2 and MMP9, which was inhibited by silence of circDENND4C. We hypothesized that anti-metastatic role of circDENND4C silence might be associated with epithelial mesenchymal transition, which needs further study in future. These indicated circDENND4C as a therapeutic target for breast cancer patients.

Prior studies have revealed that circRNAs could serve as sponges of miRNAs [27-29]. To figure out whether circDENND4C regulates breast cancer progression by sponging miRNAs, its targets were explored by bioinformatics analysis using starBase, predicting that miR-200b and miR-200c might be sponged by circDENND4C, which was confirmed by luciferase activity and RIP assays. Our research showed that miR-200b and miR-200c expression levels were decreased in breast cancer cells after exposure of hypoxia, uncovering that they might be associated with regulation of hypoxia. Previous studies demonstrated that miR200b and miR-200c could inhibit glycolysis in cancers by targeting lactate dehydrogenase $A$ and sirtuin 2 [30, 31]. Additionally, miR-200b and miR-200c could suppress migration and invasion of breast cancer cells by regulating ezrin-radixin-moesin and fucosyltransferase-4 [32, 33]. These reports suggested the anti-cancer roles of $\mathrm{miR}-200 \mathrm{~b}$ and $\mathrm{miR}-200 \mathrm{c}$ by decreasing glycolysis, migration and invasion in human cancers. The current study using rescue experiments revealed that knockdown of miR-200b and miR-200c attenuated the anti-cancer role of circDENND4C silence in breast cancer under hypoxia, indicating that circDENND4C regulates breast cancer progression by sponging miR-200b and miR-200c. Besides, in vivo experiments further supported the suppressive effect of circDENND4C interference by increasing miR$200 \mathrm{~b}$ and miR-200c. The function of miRNAs is realized by regulating mRNA expression, hence the promising targets of miR-200b and miR-200c should be explored in further study. Besides, hypoxia is regulated not only by $\mathrm{HIF} 1 \mathrm{~A}$, but also via mTORC1 signaling. Here we found that knockdown of circDENND4C suppressed the activation of p70S6K1, a marker of mTORC1 signaling (Additional file 11: Figure $\mathrm{S} 11 \mathrm{~A}$ and $11 \mathrm{~B}$ ), indicating the importance of this pathway for mechanism mediated by circDENND4C. While the more details need further study in future. 


\section{Conclusion}

In summary, this study highlighted circDENND4C as a novel prognostic target for breast cancer and elucidated that knockdown of circDENND4C plays an anti-cancer role through suppressing glycolysis, migration and invasion in breast cancer under hypoxia, possibly by increasing miR-200b and miR-200c. This work provides a new mechanism for understanding cancer progression under hypoxia and indicates circDENND4C as a promising therapeutic target for breast cancer.

\section{Additional files}

Additional file 1: Figure S1. The scheme of circDENND4C. (TIF 392 kb)

Additional file 2: Figure S2. The pathology of breast cancer tissues was analyzed by hematoxylin and eosin (HE) staining. (TIF $853 \mathrm{~kb}$ )

Additional file 3: Figure S3. The levels of circDENND4C and miR-200b/ C in breast cancer. (A-C) The expressions of circDENND4C (A), miR-200b (B) and miR-200c (C) were detected in tumor tissues and normal tissues by chromogenic in-situ hybridization assay. (TIF 3091 kb)

Additional file 4: Figure S4. The expression of circDENND4C in different types of breast cancer. ${ }^{* *} P<0.001$. (TIF $111 \mathrm{~kb}$ )

Additional file 5: Figure S5. The expression of circDENND4C in breast cancer cells by chromogenic in-situ hybridization assay. (TIF 2269 kb)

Additional file 6: Figure S6. The expression of HIF1A in breast cancer cells after treatment of hypoxia. (A and B) The protein level of HIF1A was detected in MDA-MB-453 and SK-BR-3 cells after treatment of hypoxia for $0,3,6,12,24$ and 48 h. ${ }^{*} P<0.05,{ }^{* *} P<0.01,{ }^{* * *} P<0.001$. (TIF $276 \mathrm{~kb}$ )

Additional file 7: Figure S7. The effect of HIF1A on circDENND4C level. ( $A$ and $B$ ) The protein level of HIF1A was measured in MDA-MB-453 and SK-BR-3 cells with transfection of si-NC or si-circ and treatment of hypoxia. circDENND4C expression was detected in MDA-MB-453 and SK-BR-3 cells transfected with si-HIF1A under normoxia ( $C$ and D) or si-HIF1A under hypoxia (E and F). ${ }^{*} P<0.05,{ }^{* *} P<0.01$, ${ }^{* *} P<0.001$. (TIF $727 \mathrm{~kb}$ )

Additional file 8: Figure S8. The effect of circDENND4C on migration and invasion of breast cancer cells under normoxia. circDEND4C expression (A), migration (B) and invasion (C) were detected in MDA-MB-453 and SK-BR-3 cells transfected with pcDNA or circDENND4C under normoxia. ${ }^{* *} P<0.01,{ }^{* *} P<0.001$, NS: not significant. (TIF 208 kb)

Additional file 9: Figure $\mathbf{5 9}$. The validation of stable silencing system in MDA-MB-453 cells. MDA-MB-453 cells were transfected with sh-NC, sh-circ or empty, and then the expression of circDENND4C (A) and infection efficiency (B) were analyzed by qRT-PCR or fluorescence microscope. ${ }^{* * *} P<0.001$. (TIF $961 \mathrm{~kb}$ )

Additional file 10: Figure S10. The expression of HIF1A in xenograft model by immunohistochemistry. (TIF 854 kb)

Additional file 11: Figure S11. The effect of circDENND4C on mTORC1 signaling. (A and B) The protein levels of p-p70S6K1 and p7056K1 were detected in MDA-MB-453 and SK-BR-3 cells transfected with si-NC or si-circ after treatment of hypoxia. (TIF $301 \mathrm{~kb}$ )

\section{Abbreviations}

circDENND4C: circRNA differentially expressed in normal cells and neoplasia domain containing 4C; MMP9: Matrix metallopeptidase 9; qRT-PCR: Quantitative real-time polymerase chain reaction; RIP: RNA Immunoprecipitation; si-NC: siRNA negative control; siRNA: Small interfering RNA; SOCS3: Suppressor of cytokine signaling 3

Acknowledgements

Not applicable.

\section{Authors' contributions}

conceptualization: SR and LL; Methodology: YF; Formal analysis: LH; Data curation: ZL; Software: YZ; Validation: $J$ and XC; Investigation: YF and Z; Writing - original draft preparation: SR and JL; Writing - review and editing: $S R$ and $J$; Approval of final manuscript: all authors.

\section{Funding}

This work was supported by the Key Scientific Research Project of Henan Higher Educational Institutions (Grant No. 16A320064).

Availability of data and materials

Please contact author for data request.

Ethics approval and consent to participate

The authors declare that the data supporting the findings of this study are available within the article. The manuscript was approved by the Third Affiliated Hospital of Zhengzhou University and was in accordance with the national guidelines for care and maintenance of laboratory animals.

\section{Consent for publication}

All authors consent this manuscript to be published.

\section{Competing interests}

The authors declare that they have no competing interests.

\section{Author details}

${ }^{1}$ Department of Thyroid and Breast Surgery, the Luoyang Central Hospital Affiliated to Zhengzhou University, No. 288 Zhongzhou Middle Road, Xigong District, Luoyang, China. ${ }^{2}$ Department of Breast Surgery, the Third Affiliated Hospital of Zhengzhou University, No. 7 Kangfuqian Street, Erqi District, Zhengzhou 450052, China.

Received: 29 May 2019 Accepted: 27 August 2019

Published online: 05 September 2019

References

1. Harbeck N, Gnant M. Breast cancer. Lancet. 2017;389(10074):1134-50.

2. Semenza GL. The hypoxic tumor microenvironment: a driving force for breast cancer progression. Biochim Biophys Acta. 2016;1863(3):382-91.

3. Huang R, Zong X. Aberrant cancer metabolism in epithelial-mesenchymal transition and cancer metastasis: mechanisms in cancer progression. Crit Rev Oncol Hematol. 2017;115:13-22.

4. Ojha R, Nandani R, Chatterjee N, Prajapati VK. Emerging role of circular RNAs as potential biomarkers for the diagnosis of human diseases. Adv Exp Med Biol. 2018;1087:141-57.

5. Zhang HD, Jiang LH, Sun DW, Hou JC, Ji ZL. CircRNA: a novel type of biomarker for cancer. Breast Cancer. 2018;25(1):1-7.

6. Tomar D, Yadav AS, Kumar D, Bhadauriya G, Kundu GC. Non-coding RNAs as potential therapeutic targets in breast cancer. Biochim Biophys Acta Gene Regul Mech. 2019. https://doi.org/10.1016/j.bbagrm.2019.04.005.

7. Xu JZ, Shao CC, Wang XJ, Zhao X, Chen JQ, Ouyang YX, Feng J, Zhang F, Huang WH, Ying Q, Chen CF, Wei XL, Dong HY, Zhang GJ, Chen M. circTADA2As suppress breast cancer progression and metastasis via targeting miR-203a-3p/SOCS3 axis. Cell Death Dis. 2019;10(3):175.

8. Zhang HD, Jiang LH, Hou JC, Zhong SL, Zhou SY, Zhu LP, Li J, Wang DD, Sun DW, Ji ZL, Tang JH. Circular RNA hsa_circ_0052112 promotes cell migration and invasion by acting as sponge for miR-125a-5p in breast cancer. Biomed Pharmacother. 2018;107:1342-53.

9. Boeckel JN, Jae N, Heumuller AW, Chen W, Boon RA, Stellos K, Zeiher AM, John D, Uchida S, Dimmeler S. Identification and characterization of hypoxia-regulated endothelial circular RNA. Circ Res. 2015;117(10):884-90.

10. Liang G, Liu Z, Tan L, Su AN, Jiang WG, Gong C. HIF1alpha-associated circDENND4C promotes proliferation of breast Cancer cells in hypoxic environment. Anticancer Res. 2017;37(8):4337-43.

11. Bach DH, Lee SK, Sood AK. Circular RNAs in Cancer. Mol Ther Nucleic Acids. 2019;16:118-29.

12. Nassar FJ, Nasr R, Talhouk R. MicroRNAs as biomarkers for early breast cancer diagnosis, prognosis and therapy prediction. Pharmacol Ther. 2017;172:34-49. 
13. Yao Y, Hu J, Shen Z, Yao R, Liu S, Li Y, Cong H, Wang X, Qiu W, Yue L. MiR-200b expression in breast cancer: a prognostic marker and act on cell proliferation and apoptosis by targeting Sp1. J Cell Mol Med. 2015;19(4):760-9.

14. Xu F, He H, Huang W, Lin Y, Luo S, Du Q, Duan R. Decreased expression of MicroRNA-200 family in human breast cancer is associated with lymph node metastasis. Clin Transl Oncol. 2016;18(3):283-8.

15. Chang BP, Wang DS, Xing JW, Yang SH, Chu Q, Yu SY. miR-200c inhibits metastasis of breast cancer cells by targeting HMGB1. J Huazhong Univ Sci Technolog Med Sci. 2014;34(2):201-6.

16. Zhang T, Wan JG, Liu JB, Deng M. MiR-200c inhibits metastasis of breast tumor via the downregulation of Foxf2. Genet Mol Res. 2017;16(3). https:// doi.org/10.4238/gmr16038971.

17. Bian X, Liang Z, Feng A, Salgado E, Shim H. HDAC inhibitor suppresses proliferation and invasion of breast cancer cells through regulation of miR-200c targeting CRKL. Biochem Pharmacol. 2018;147:30-7.

18. Livak KJ, Schmittgen TD. Analysis of relative gene expression data using real-time quantitative PCR and the 2(-Delta Delta $C(T))$ method. Methods. 2001;25(4):402-8.

19. Garcia SN, Guedes RC, Marques MM. Unlocking the potential of HK2 in cancer metabolism and therapeutics. Curr Med Chem. 2018. https://doi. org/10.2174/0929867326666181213092652.

20. Shi T, Ma Y, Cao L, Zhan S, Xu Y, Fu F, Liu C, Zhang G, Wang Z, Wang R, Lu H, Lu B, Chen W, Zhang X. B7-H3 promotes aerobic glycolysis and chemoresistance in colorectal cancer cells by regulating HK2. Cell Death Dis. 2019;10(4):308

21. Jiang S, Zhang LF, Zhang HW, Hu S, Lu MH, Liang S, Li B, Li Y, Li D, Wang ED, Liu MF. A novel miR-155/miR-143 cascade controls glycolysis by regulating hexokinase 2 in breast cancer cells. EMBO J. 2012;31(8):1985-98,

22. Gao T, Li JZ, Lu Y, Zhang CY, Li Q, Mao J, Li LH. The mechanism between epithelial mesenchymal transition in breast cancer and hypoxia microenvironment. Biomed Pharmacother. 2016;80:393-405.

23. Joseph JP, Harishankar MK, Pillai AA, Devi A. Hypoxia induced EMT: a review on the mechanism of tumor progression and metastasis in OSCC. Oral Oncol. 2018;80:23-32.

24. Alaseem A, Alhazzani K, Dondapati P, Alobid S, Bishayee A, Rathinavelu A. Matrix metalloproteinases: a challenging paradigm of cancer management. Semin Cancer Biol. 2019;56:100-15.

25. Jiang $Q$, Pan Y, Cheng Y, Li H, Liu D, Li H. Lunasin suppresses the migration and invasion of breast cancer cells by inhibiting matrix metalloproteinase-2/ -9 via the FAK/Akt/ERK and NF-kappaB signaling pathways. Oncol Rep. 2016;36(1):253-62.

26. Abdullah ML, Hafez MM, Al-Hoshani A, Al-Shabanah O. Anti-metastatic and anti-proliferative activity of eugenol against triple negative and HER2 positive breast cancer cells. BMC Complement Altern Med. 2018;18(1):321.

27. Verduci L, Strano S, Yarden Y, Blandino G. The circRNA-microRNA code: emerging implications for cancer diagnosis and treatment. Mol Oncol. 2019;13(4):669-80

28. Yan L, Zheng M, Wang H. Circular RNA hsa_circ_0072309 inhibits proliferation and invasion of breast cancer cells via targeting miR-492. Cancer Manag Res. 2019;11:1033-41.

29. Yang L, Song C, Chen Y, Jing G, Sun J. Circular RNA circ_0103552 forecasts dismal prognosis and promotes breast cancer cell proliferation and invasion by sponging miR-1236. J Cell Biochem. 2019;120(9):15553-60.

30. Hu S, Jiang Q, Luo D, Zhao L, Fu X, Chen Y, Song X, Li L, Zhao H, He Y, Peng B. miR-200b is a key regulator of tumor progression and metabolism targeting lactate dehydrogenase A in human malignant glioma. Oncotarget. 2016;7(30):48423-31.

31. Cha Y, Han MJ, Cha HJ, Zoldan J, Burkart A, Jung JH, Jang Y, Kim CH, Jeong HC, Kim BG, Langer R, Kahn CR, Guarente L, Kim KS. Metabolic control of primed human pluripotent stem cell fate and function by the miR-200CSIRT2 axis. Nat Cell Biol. 2017;19(5):445-56.

32. Hong H, Yu H, Yuan J, Guo C, Cao H, Li W, Xiao C. MicroRNA-200b impacts breast Cancer cell migration and invasion by regulating Ezrin-radixinMoesin. Med Sci Monit. 2016:22:1946-52.

33. Jurmeister S, Baumann M, Balwierz A, Keklikoglou I, Ward A, Uhlmann S, Zhang JD, Wiemann S, Sahin O. MicroRNA-200c represses migration and invasion of breast cancer cells by targeting actin-regulatory proteins FHOD1 and PPM1F. Mol Cell Biol. 2012;32(3):633-51.

\section{Publisher's Note}

Springer Nature remains neutral with regard to jurisdictional claims in published maps and institutional affiliations.
Ready to submit your research? Choose BMC and benefit from:

- fast, convenient online submission

- thorough peer review by experienced researchers in your field

- rapid publication on acceptance

- support for research data, including large and complex data types

- gold Open Access which fosters wider collaboration and increased citations

- maximum visibility for your research: over $100 \mathrm{M}$ website views per year

At BMC, research is always in progress.

Learn more biomedcentral.com/submissions 